\title{
Exhaust-gas imaging via planar laser- induced fluorescence of sulfur dioxide
}

The University of Michigan, Department of Mechanical Engineering, 2202 GG Brown Building, 2350 Hayward Street, Ann Arbor, MI 48109-2125, USA

Received: 14 January 2002/Revised version: 30 January 2002

Published online: 14 March 2002 • (C) Springer-Verlag 2002

ABSTRACT The potential use of planar laser-induced fluorescence (PLIF) of sulfur dioxide $\left(\mathrm{SO}_{2}\right)$ for visualization of exhaust-gas distributions is outlined and demonstrated. Strong absorption features in the UV spectral range allow excitation of $\mathrm{SO}_{2}$ with the fourth harmonic of a Nd:YAG laser at $266 \mathrm{~nm}$. Fluorescence emissions are mostly red-shifted and can be easily detected in single-shot imaging arrangements with a good signal-to-noise ratio. This study uses a premixed methane/air flame that is doped with $\mathrm{SO}_{2}$ to demonstrate the technique. The signal strength has a pronounced temperature dependence for excitation at $266 \mathrm{~nm}$.

PACS 33.20.Lg; 39.30.+w; 42.62.Fi

1

\section{Introduction}

The operation of reciprocating internal combustion engines with the characteristic cyclic charging, burning, and exhausting steps inevitably is associated with some of the burnt charge remaining inside the cylinder after the exhaust stroke. The mixing of the fresh charge with this remaining residual exhaust gas can have a significant impact on the combustion. Especially, with newer types of engines, like gasoline direct injection or homogeneous charge compression ignition engines, complete mixing of residual gas or exhaust gas recirculation (EGR) gases with the fresh charges might not be obtained due to particular timing issues with fuel injection and valve operation. This can lead to substantial cyclic variability in the spatial distribution of the fuel/air/residual ratios and therefore erratic operation with fluctuating power output and increased pollutant emissions can be the result. In the past decade, improvements of in-cylinder fuel/air mixing concepts have been sub- stantially aided by the use of laser-based imaging techniques that visualize the fuel concentration or equivalence ratio directly in the cylinder of an engine that is modified for optical access. Most often, these studies use single-component fuels, like iso-octane, and a fluorescent tracer, like diethylketone [1] or toluene [2]. With such an approach it was possible to quantify the laserinduced fluorescence signals and to determine distributions of number densities or equivalence ratios. The direct imaging of exhaust gas would require us to measure water or carbon dioxide distributions: the two molecules are major constituents of exhaust gas. Water can be detected via a two-photon laserinduced fluorescence (LIF) scheme with excitation at $248 \mathrm{~nm} \mathrm{[3],} \mathrm{but} \mathrm{the} \mathrm{two-}$ photon excitation makes it difficult to apply for quantitative measurements under engine conditions. Infrared LIF was successfully demonstrated for carbon dioxide [4]. High-pressure applications in hot enclosures seem a difficult environment for this approach. Thus, an alternative imaging strategy for the vi- sualization of burnt gases is needed and a suitable tracer must be found for this. This paper proposes the use of sulfur dioxide $\left(\mathrm{SO}_{2}\right)$ as a suitable tracer for imaging diagnostics of exhaust gases. $\mathrm{SO}_{2}$ is not naturally present in the unburnt gases - at least for frequently used model fuels - and therefore almost any sulfur-containing precursor (hydrogen sulfide, mercaptans, thiophene, etc.) that is added to the fuel will be oxidized in the flame to yield $\mathrm{SO}_{2}$ that then can be detected via laser-induced fluorescence. The $\widetilde{A}^{1} B_{1}-\widetilde{X}^{1} A_{1}$ system in the UV spectral range is a good candidate to devise a new imaging strategy, as several common laser sources in this range can be used for excitation. We describe the use of a frequency-quadrupled Nd:YAG laser. In earlier work, Muller et al. [5] have used excitation of $\mathrm{SO}_{2}$ in the same electronic system for point-wise measurements to study sulfur chemistry in flames.

\section{$2 \quad$ Experimental setup}

Laser-induced fluorescence of $\mathrm{SO}_{2}$ upon excitation at $266 \mathrm{~nm}$ was studied in a premixed methane/air flame of a simple laboratory Bunsen burner with a nozzle opening diameter of $10 \mathrm{~mm}$. $\mathrm{SO}_{2}$ could be added to the methane, with both gas flows being controlled by mass-flow controllers (Tylan). Spectroscopic degrees of freedom to be evaluated for a new detection scheme include excitation and emission wavelengths. This work focuses on wavelengths near $266 \mathrm{~nm}$, the fourth harmonic of Nd:YAG lasers. A tunable version of a single-rod Nd:YAG laser was used (LaVision T-YAG) that could deliver up to $65 \mathrm{~mJ}$ of $266-\mathrm{nm}$ 
light per pulse. The tuning range covers the small range between the main band $(1064.04 \mathrm{~nm})$ and the side band $(1064.60 \mathrm{~nm})$, but this is potentially enough to selectively excite molecules with a pronounced rotational structure.

Signals were collected at right angles to the laser beam with an $f_{\#}=$ 2 UV-transparent lens and dispersed in a $150-\mathrm{mm}$-spectrograph (ARC-150) that was equipped with two gratings, $300 \mathrm{l} / \mathrm{mm}$ and $1200 \mathrm{l} / \mathrm{mm}$; both blazed for $300 \mathrm{~nm}$. The dispersed LIF signals were detected with a 14-bit ICCD camera (LaVision FlameStar IIF). Instantaneous PLIF images of $\mathrm{SO}_{2}$ were taken with the same camera, the same UV lens, and a WG 280 Schott glass filter that rejected Rayleigh scattering at $266 \mathrm{~nm}$. Laser intensities of $2.5 \mathrm{MW} / \mathrm{cm}^{2}$ were sufficient to produce high-quality single-shot images at seeding levels of approximately $3000-\mathrm{ppm} \mathrm{SO}_{2}$. All investigations that are discussed here use $\mathrm{SO}_{2}$-doped flames rather than producing $\mathrm{SO}_{2}$ from a precursor as discussed above. This eliminates ambiguities in the assignment of spectral features and facilitates the interpretation of the results at this stage of the development. In addition, spectra were recorded with the gas mixture (methane and $\mathrm{SO}_{2}$ ) flowing through the burner, but not ignited, to address some issues of temperature dependence in the LIF signals.

\section{Results}

Spectra were taken after excitation in the post-flame region of the premixed flame where no large temperature and concentration gradients are present. As expected, the images that were recorded behind the spectrograph did not show any spatial variation and, therefore, the emission spectra that were measured in this region were averaged over the spatial coordinate and only single-line spectra are reported here. A non-reacting flow of methane/air $/ \mathrm{SO}_{2}$ was investigated at the same location. The LIF emission spectra of the room-temperature, atmospheric pressure methane/air/ $\mathrm{SO}_{2}$ mixture after excitation at $266 \mathrm{~nm}$ are shown in Fig. 1. For this, as well as the combusting case, no excitation-wavelength dependence was noticed over the small tuning range of the Nd:YAG laser.

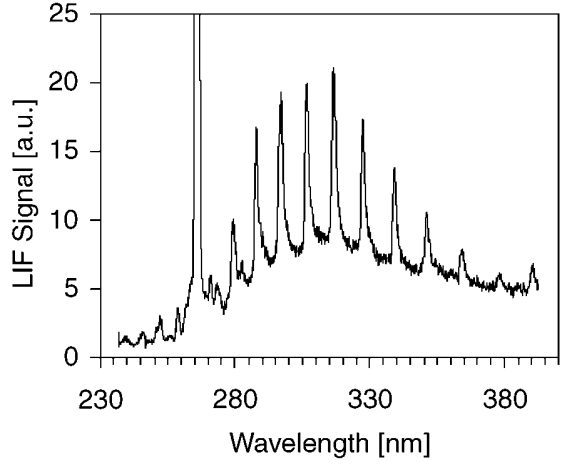

FIGURE 1 LIF emission spectra of $\mathrm{SO}_{2}$ room temperature and atmospheric pressure after excitation at $266 \mathrm{~nm}$. Dispersion was achieved with a 150-mm imaging spectrograph, an entrance slit of $100 \mu \mathrm{m}$, and a $1200 \mathrm{l} / \mathrm{mm}$ grating blazed for $300 \mathrm{~nm}$. The intense peak at $266 \mathrm{~nm}$ is Rayleigh scattering

A very distinct vibrational structure is visible with an average line spacing of approximately $1050 \mathrm{~cm}^{-1}$. The three vibrational modes of $\mathrm{SO}_{2}$ have distinctly different vibrational frequencies, none of which is close to the observed line spacing. However, as pointed out by Herzberg [6, pp. 150 ff.], involvement of an antisymmetric vibration would lead to a selection rule where $\Delta v= \pm 2, \pm 4$ etc., and one expects to find emission with every second band missing. (See also [6, p. 512] and footnotes therein on the complexity of the $\mathrm{SO}_{2}$ spectra.) Thus, the spacing is in agreement with twice the vibrational frequency of the $v_{2}^{\prime \prime}$ bending mode $\left(v_{2}^{\prime \prime}=517 \mathrm{~cm}^{-1}\right)$. The observed transitions at wavelengths shorter than the excitation wavelength point to the fact that the excitation started at a higher vibrational level of the electronic ground state. Vibrational levels $v_{2}^{\prime}$ of up to $v_{2}^{\prime \prime}=45$ can be excited with $266 \mathrm{~nm}$ when starting from the ground state. A vibrational level of less than $v_{2}^{\prime}=38$ is not in agreement with the observations, as this would not allow us to produce the emissions at the shortest wavelengths. A best match of the measured line positions is found for $v_{2}^{\prime}=44,41$, and 38 . This would mean that excitation started at $v_{2}^{\prime \prime}=11$ or 12,9 or 10 , and 8 , respectively. Weaker lines at wavelengths less than $300 \mathrm{~nm}$ belong to a different progression.

Figure 2 illustrates the pronounced difference of the LIF spectra of $\mathrm{SO}_{2}$ at higher temperatures $(\sim 1800 \mathrm{~K})$. The signal intensity increased by up to a factor of 30 at some emission wavelengths. The Rayleigh peak at $266 \mathrm{~nm}$ barely

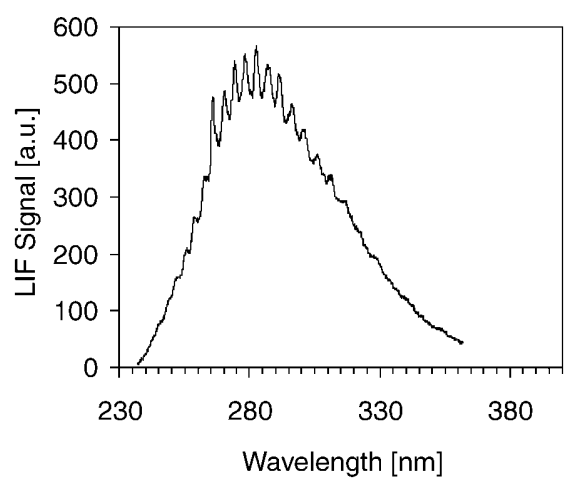

FIGURE 2 LIF emission spectra of $\mathrm{SO}_{2}$ in a premixed methane/air flame at atmospheric pressure after excitation at $266 \mathrm{~nm}$. Dispersion was achieved with a $150-\mathrm{mm}$ imaging spectrograph, an entrance slit of $100 \mu \mathrm{m}$, and a $12001 / \mathrm{mm}$ grating blazed for $300 \mathrm{~nm}$

stands out beyond the LIF signals. Not only did the LIF signal intensity increase substantially, but also many more transitions are now visible. The line spacing again points to transitions caused by the $v_{2}$ bending mode, now originating from other vibrational states.

The transitions at the longer wavelengths start from lower vibrational states in absorption. Thus, it is to be expected that the strong temperature dependence of the LIF signals that is observed after excitation at $266 \mathrm{~nm}$ will decrease when exciting at longer wavelengths. Suitable laser sources (besides tunable sources like dye lasers and OPOs) would be $\mathrm{XeCl}(308 \mathrm{~nm})$, $\mathrm{XeF}(351 \mathrm{~nm})$, or the third harmonic of $\mathrm{Nd}$ :YAG $(355 \mathrm{~nm})$ lasers.

Reshaping of the 266-nm laser beam into a light sheet of approximately 1-mm thickness and a power density of $2.5 \mathrm{MW} / \mathrm{cm}^{2}$ was achieved with a combination of three lenses. This sheet then illuminated a vertical plane through the center axis in the flame starting at about $5 \mathrm{~mm}$ above the nozzle exit. An example of a single-pulse image from a flame where the methane was doped with 3000 ppm of $\mathrm{SO}_{2}$ is shown in Fig. 3. The image has not been corrected for spatial variations of energy across the laser sheet. These are in particular visible in the bright streak in the upper part of Fig. 3.

The inner cone where the unburnt fuel/ $\mathrm{SO}_{2}$ /air mixture is still cold is visualized as a dark area with low LIF intensity according to the finding discussed above. There are measurable sig- 


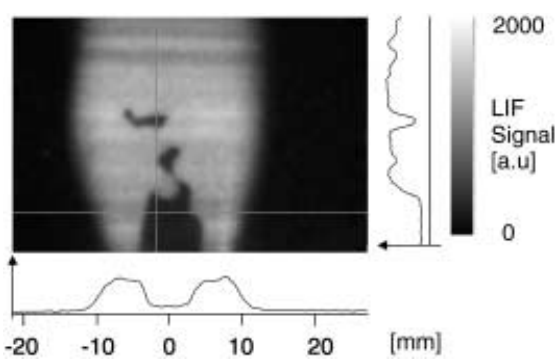

FIGURE 3 Single-pulse PLIF image of $\mathrm{SO}_{2}$ in a premixed methane/air flame. The gray lines in the image indicate the position of the profiles that are shown on the right of and below the image

nals substantially above the noise level, as seen in the horizontal profile across the flame. Note that this flame is not spatially steady and that the inner cone fluctuates. This produces pockets of unburnt fuel/air mixture, which are also seen in Fig. 3.

\section{$4 \quad$ Conclusions and open issues}

It was successfully demonstrated that small amounts of $\mathrm{SO}_{2}$ in flame gases can be imaged on a singlepulse basis when excited with the fourth harmonic of a Nd:YAG laser. The vibrational structure of the laser-inducedfluorescence emission has been identified and explains the strong temperature dependence of the fluorescence signal. A more precise assignment of the vibrational progressions will be possible through measurements at various fixed temperatures, when the temperaturedependent signal can be related to the partition functions.

Future investigations will address strategies to minimize the temperature dependence of the LIF signals to develop a quantitative single-line imaging scheme for exhaust gas recirculation and residual gases. This primarily means the use of other excitation sources, like $\mathrm{XeCl}, \mathrm{XeF}$, or tripled Nd:YAG lasers. Alternatively, multiline schemes will be examined to extract temperature and concentration information from $\mathrm{SO}_{2}$ PLIF images. It will also be necessary to investigate the fluorescence-quenching properties of $\mathrm{SO}_{2}$ over a wide range of temperatures and for a range of collider molecules. Also, a range of precursors that will produce $\mathrm{SO}_{2}$ in flames will have to be tested.

ACKNOWLEDGEMENTS This work was sponsored by the General Motors Corporation through the General Motors Collaborative Research Laboratory at the University of Michigan. The assistance of Stephen L. Yee during the initial experiments is acknowledged.

\section{REFERENCES}

1 S. Einecke, C. Schulz, V. Sick: Appl. Phys. B 71, 717 (2000)

2 J. Reboux, D. Puechberty, F. Dionnet: SAE Tech. Pap. Ser. 941988 (1994)

3 R.W. Pitz, T.S. Cheng, J.A. Wehrmeyer, C.F. Hess: Appl. Phys. B 56, 94 (1993)

4 B.J. Kirby, R.K. Hanson: Appl. Phys. B 69, 505 (1999)

5 C.H. Muller III, K. Schofield, M. Steinberg, H.P. Broida: Proc. Combust. Inst. 17, 867 (1979)

6 G. Herzberg: Molecular Spectra and Molecular Structure, Vol. III (Krieger, Malabar, FL 1991) 